\title{
Role of Angiotensin III in Hypertension
}

\author{
Annabelle Reaux-Le Goazigo, PhD, Xavier Iturrioz, PhD, Celine Fassot, PhD, \\ Cedric Claperon, PharmG, Prof. Bernard P. Roques, \\ and Catherine Llorens-Cortes, $\mathrm{PhD}$
}

\author{
Address \\ INSERM 69I-Collège de France, II Place Marcelin Berthelot, \\ 7523I Paris Cedex 05, France. \\ E-mail: c.llorens-cortes@college-de-france.fr
}

The hyperactivity of the brain renin-angiotensin system (RAS) has been implicated in the development and maintenance of hypertension in several types of experimental and genetic hypertension animal models. Among the main bioactive peptides of the brain RAS, angiotensin (Ang) II and Ang III display the same affinity for type I and type 2 Ang II receptors. Both peptides, injected intracerebroventricularly, similarly increase blood pressure (BP); however, because Ang II is converted in vivo to Ang III, the identity of the true effector is unknown. In this article, we review new insights into the predominant role of brain Ang III in the control of BP, underlining the fact that brain aminopeptidase A (APA), the enzyme-forming central Ang III, could constitute a putative central therapeutic target for the treatment of hypertension. This justifies the development of potent systemically active APA inhibitors, such as RBI50, as prototypes of a new class of antihypertensive agents for the treatment of certain forms of hypertension.

\section{Introduction}

Hypertension is a major cardiovascular risk factor affecting approximately $10 \%$ of the population. Most (95\%) have essential hypertension of uncertain etiology. The implication of a central component in animal models and in humans has been suggested, and may be the origin of sympathetic hyperactivity observed in the early stages of this pathology [1].

The hyperactivity of the brain renin-angiotensin system (RAS) has been implicated in the development and maintenance of hypertension in several types of experimental and genetic hypertension animal models, such as spontaneously hypertensive rats (SHR), deoxycorticosterone acetate (DOCA)-salt hypertensive rats [2,3], and transgenic animals harboring the mouse renin ren $2^{d}$ gene $[4,5]$ or overexpressing both human angiotensinogen and human renin $[6,7]$. The activity of the systemic RAS is normal in the SHR model, depressed in DOCA-salt rats, and high in transgenic animals.

All the components of the RAS, including the precursor and enzymes required for the production and degradation of angiotensins and specific angiotensin receptor type 1 $\left(\mathrm{AT}_{1}\right)$ and type $2\left(\mathrm{AT}_{2}\right)$, have been identified in the brain $[8,9]$. By analogy with the systemic RAS, brain Ang II is generated by sequential cleavage of the precursor, angiotensinogen, by an aspartyl protease, renin, producing the inactive decapeptide angiotensin I (Asp-Arg-Val-Tyr-IleHis-Pro-Phe-His-Leu), which is then converted to Ang II by a membrane-bound zinc-metalloprotease, angiotensinconverting enzyme (ACE). Ang II is then metabolized to generate Ang III, which is itself converted to Ang IV by aminopeptidases. Two of the effector peptides of the brain RAS, Ang II and Ang III, display similar affinities for $\mathrm{AT}_{1}$ and $\mathrm{AT}_{2}$ receptors $[10,11]$. When injected into the brain, these peptides interact with $\mathrm{AT}_{1}$ receptors, similarly increasing blood pressure (BP), drinking behavior, salt appetite, and pituitary hormone release $[12,13]$.

Ang II was thought to be the principal active central angiotensin peptide. However, Ang II is converted in vivo into its direct metabolite Ang III, and several studies suggest that Ang III may be the true effector of the brain RAS in the control of BP [14-16]. If this is the case, then selectively blocking the formation of brain Ang III should decrease BP. Thus, the enzyme that generates Ang III might be a potential target for the treatment of hypertension.

In this article, we summarize what is currently known about the molecular and biochemical properties of aminopeptidase A (APA), which constitutes, together with aminopeptidase N (APN), the two enzymes involved in the in vivo metabolism of brain Ang II and Ang III, respectively. The development of specific and selective APA and APN inhibitors has subsequently allowed selective blocking of the metabolic pathways of brain Ang II and Ang III and investigation into the respective roles of these two peptides in the control of BP.

\section{Exploration of the Aminopeptidase A Active Site: Modeling of Aminopeptidase A Site-directed mutagenesis studies \\ In the absence of structural data on monozinc aminopepti- dases, the organization of the APA active site was investigated}




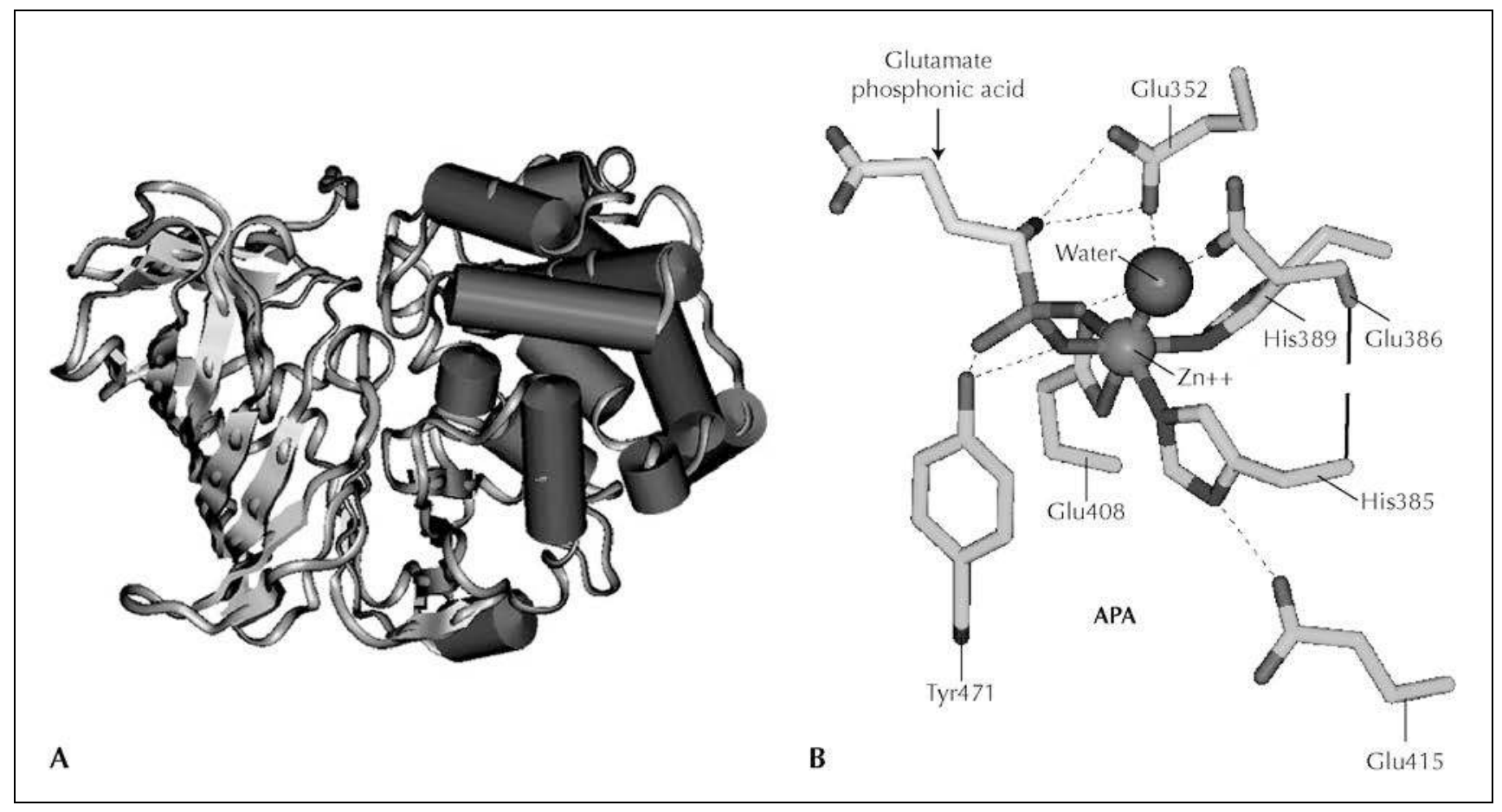

Figure 1. Homology modeling of APA, using $\mathrm{LTA}_{4} \mathrm{H}$ as a template. A, Ribbon diagram of the tertiary structure of APA. The protein is organized into three domains: 1) the $\mathrm{N}$-terminal domain, which consists mostly of $\beta$ sheets (left); 2 ) the globular active-site domain; and 3) the C-terminal helical domain (cylinders). B, Model of the inhibitor glutamate phosphonate docked into the APA active site. The zinc atom $\left(\mathrm{Zn}^{++}\right)$is shown as a small sphere and the water molecule as a large sphere. The $\mathrm{Zn}$-ligand interactions are shown as thick lines; the hydrogen bonds between the active site residues and the water molecule and between the active site residues and the inhibitor are shown in dashed lines. APA—aminopeptidase A.

by means of site-directed mutagenesis. In 1993, Wang and Cooper [17] identified histidine 389, in the HEXXH (385389) motif of APA, as one of the three zinc ligands. Glu 408, in the WLNEG (405-409) motif of APA, is conserved in the various monozinc aminopeptidases and located at a constant distance from the HEXXH motif. Vazeux et al. [18] showed that this residue constitutes the third zinc ligand and that glutamate (Glu) 386, located in the zinc-binding HEXXH motif, plays a critical role in catalysis as the catalytic effector in APA. Alignment of the sequence of APA surrounding the zincbinding motif with those of several monozinc aminopeptidases led to the identification of a conserved tyrosine residue (Tyr 471 in APA). Site-directed mutagenesis of Tyr 471 demonstrated that this residue is essential for the catalytic activity of APA. This residue stabilizes the transition state complex by hydrogen bonding between the hydroxyl group of the tyrosine and the oxyanion of the tetrahedral intermediate [19]. This function is similar to those proposed for His 231 in thermolysin (TLN) and His 711 in neutral endopeptidase 24.11 (NEP) $[20,21]$. Glu 352, which is present in the conserved motif GXMEN (348-353), has been shown to be involved in the catalytic process of APA. It contributes to the exopeptidase activity of this enzyme by interacting with the $\mathrm{N}$-terminal part of the substrate [22], as shown for APN [23].

\section{Three-dimensional model of aminopeptidase A}

Rozenfeld et al. $[24 \bullet \bullet, 25]$ constructed a three-dimensional (3-D) model of residues 79 to 559 of the mouse APA ectodomain based on the functional data collected in these previous studies and the x-ray crystal structure of leukotriene $\mathrm{A}_{4}$ hydrolase $\left(\mathrm{LTA}_{4} \mathrm{H}\right)$, a bifunctional zinc metalloenzyme with both epoxide hydrolase and aminopeptidase activities [26]. This model predicts a flat, triangular structure for APA, consisting of three different domains: 1) the $\mathrm{N}$-terminal domain, consisting mostly of $\beta$ sheets; 2 ) the globular active site domain; and 3) the Cterminal helical domain (Fig. 1A). In this model, the zinc atom is coordinated by the two histidine residues (His 385 and His 389) of the HEXXH motif, together with a water molecule and Glu 408.

A potent and selective APA inhibitor 4-amino-4 phosphonobutyric acid (GluPhos) was then docked into the APA active site (Fig. 1B).

In this model, the $\mathrm{Zn}^{++}$ion is hexacoordinated:

- By three active site residues: His 385, His 389, and Glu 408,

- By one of the oxygen atoms of the phosphate of the inhibitor (reflecting the tetrahedral geometry of the peptide bond during its cleavage) and by a water molecule from the solvent (Fig. 1B).

A strong network of hydrogen bonds is kept stable around the zinc coordination sphere; the water molecule bound to the zinc ion is also engaged in two hydrogen bonds with the Glu 386 and Glu 352 side chains. This suggests that 


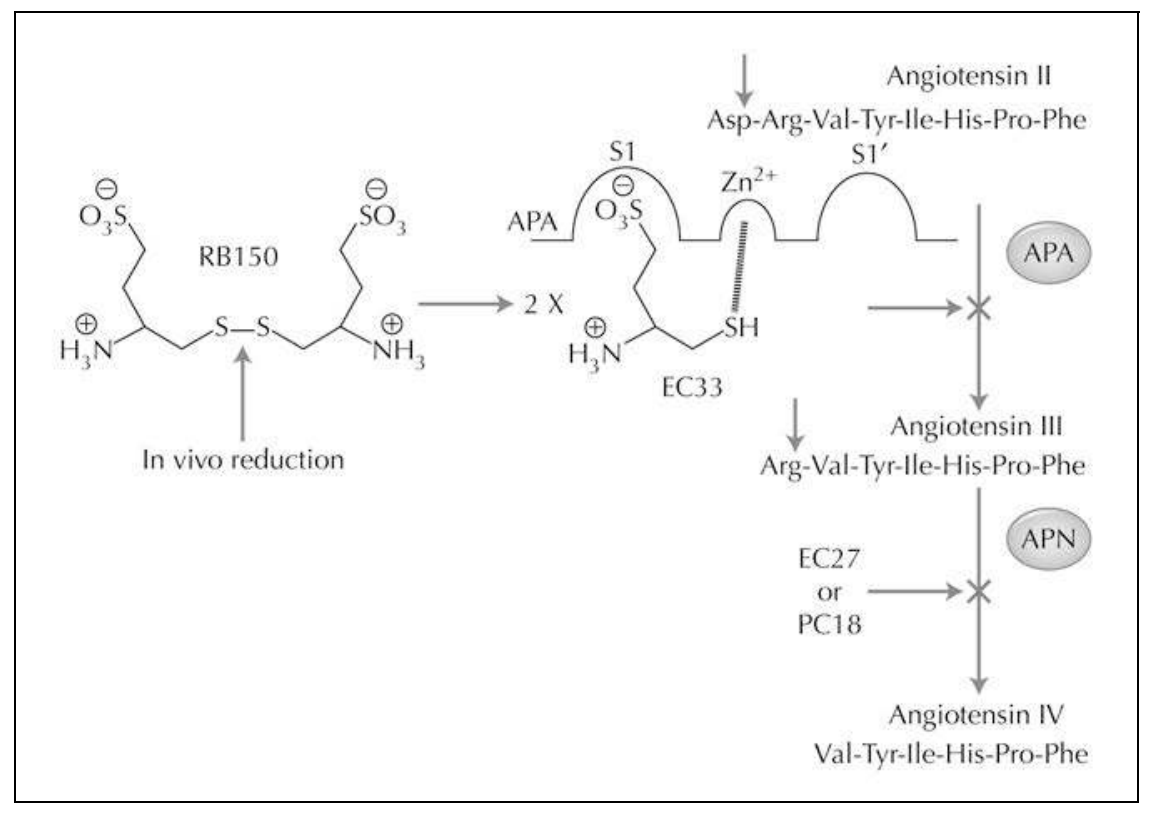

Figure 2. Metabolic pathways of Ang II and Ang III in the brain involving APA and APN. Structures of the APA inhibitor (EC33) and the corresponding prodrug (RB150). Ang-angiotensin; APA-aminopeptidase A; APN-aminopeptidase $\mathrm{N}$.

these two residues play a role in catalysis, polarizing the water molecule. The Glu 352 and Glu 215 side chains are also hydrogen-bonded to the amine moiety of the inhibitor, suggesting that these residues are responsible for the exopeptidase specificity of APA. The hydroxyl group of the phenol ring of Tyr 471 is hydrogen-bonded to an oxygen atom of the phosphate group of the inhibitor, confirming the role of this residue in stabilizing the transition state during catalysis (Fig. 1B).

This model is highly consistent with the organization of the APA-active site proposed on the basis of mutagenesis studies. The model also identifies two residues, Arg 220 and Asp 227, as playing a potentially vital structural role. They interact with each other via a salt bridge, which seems to be necessary for maintenance of the cohesion of the N-terminal $\beta$-sheet domain, which is in turn required to ensure correct folding of the N-terminal domain surrounding the active site. Site-directed mutagenesis was used to abolish the interaction between these two residues. The resulting mutated APAs were incorrectly processed and lacked enzymatic activity, confirming their key structural role and further validating the model $[24 \bullet \bullet]$. This model constitutes a powerful tool for further investigation of the active site of APA, and for designing new inhibitors of the enzyme.

\section{Design of Inhibitors of Aminopeptidases A and $\mathrm{N}$}

Before constructing the 3-D model of APA, specific and selective APA inhibitors were developed. Some of these potential inhibitors were based on the structure of the glutamate thiol [27], which is a potent but nonselective inhibitor of APA and APN, and on the similarities between the active sites of thermolysin-like enzymes [28]. Thus, Chauvel et al. [29] designed an APA inhibitor (EC33 [(S)- 3-amino-4-mercapto-butyl sulfonic acid]) in which the carboxylate of the side chain of the glutamate thiol was replaced by a sulfonate, the aim being to increase the polarity of the side chain and its interaction with the calcium ion, thus increasing selectivity toward APN. We also synthesized RB150 (4,4'-dithio bis[3-amino butyl sulfonic acid]); a systemically active prodrug of EC33 was then obtained by dimerization of EC33 through a disulfide bond (Fig. 2).

Two effective APN inhibitors were also developed: EC27 ((S)-2-amino-pentan-1,5-dithiol), which was derived from homogluthiol by replacing the side chain carboxylate group by a thiol, and PC18 (2-amino-4-methylsulfonyl butane thiol) [30] (Fig. 2). In vitro studies with purified APA and APN revealed that EC33 inhibited APA $(\mathrm{Ki}=0.29 \mu \mathrm{M})$ almost 100 times more strongly than APN, whereas EC27 inhibited APN approximately 100 times more strongly than APA $(\mathrm{Ki}=0.032 \mu \mathrm{M})$ [29]. PC18 inhibited $\mathrm{APN}(\mathrm{Ki}=0.008 \mu \mathrm{M}) 2150$ times more strongly than APA $(\mathrm{Ki}=17.2 \mu \mathrm{M})[30,31]$. The reduced form of RB150 obtained in the presence of dithiothreitol (DTT) inhibited purified APA $(\mathrm{Ki}=0.20 \pm 0.02 \mu \mathrm{M})$, similar to EC33

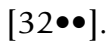

\section{Identification of the In Vivo Metabolic Pathways of Brain Ang II and Ang III}

Aminopeptidase A and APN, both of which are membrane-bound zinc-metallopeptidases [18,33,34], constituted particularly good candidates for the hydrolysis of Ang II and Ang III in vivo. This hypothesis is based on the observations that, in vitro, purified APA hydrolyzes the Nterminal aspartate of Ang II to generate Ang III [35], whereas purified APN hydrolyzes the $\mathrm{N}$-terminal arginine of Ang III to generate Ang IV [36]. 
Harding et al. [37] were the first to investigate the in vivo metabolism of brain Ang II and Ang III. They showed that the half lives of intracerebroventricularly (ICV) injected Ang II and Ang III were approximately 23 and 8 seconds, respectively. Ang III is, therefore, more rapidly metabolized than Ang II. The aminopeptidase inhibitors amastatin and bestatin were used to block the metabolism of brain Ang II and Ang III. The ICV injection of amastatin, as well as bestatin, extended the half lives of both [ $\left.{ }^{125} \mathrm{I}\right]$ Ang II and [ $\left.{ }^{125} \mathrm{I}\right]$ Ang III $[38,39]$. Although amastatin and bestatin are both efficient blockers of angiotensin metabolism, they are not selective for a given aminopeptidase. Consistent with this, amastatin, which was initially described as a specific APA inhibitor, actually inhibits APN 40 times more efficiently than it does APA, whereas bestatin displays broad specificity for various aminopeptidases $[40,41]$. For these reasons, it was difficult to draw conclusions concerning the exact nature of the aminopeptidases involved in the metabolism of brain Ang II and Ang III from these ICV experiments.

Specific and selective APA (EC33) and APN (EC27, PC18) inhibitors were subsequently used in vivo [31,42] to determine whether APA and APN hydrolyze Ang II and Ang III, respectively, in the brain. Conscious mice were injected ICV with radiolabeled angiotensins in the presence or absence of the APA or APN inhibitors. EC33 increased the half life of $\left[{ }^{3} \mathrm{H}\right]$ Ang II by a factor of 3 and completely blocked the formation of $\left[{ }^{3} \mathrm{H}\right]$ Ang III, whereas EC27 and PC18 increased the half life of $\left[{ }^{3} \mathrm{H}\right]$ Ang III by factors of 2 and 4, respectively [31,42].

These results provided the first demonstration that APA and APN are involved in vivo in the metabolism of brain Ang II and Ang III, respectively [31,42].

\section{Angiotensin III and Central Control of Blood Pressure}

Ang II and Ang III, injected separately ICV into normotensive Wistar-Kyoto (WKY) rats or SHR, cause similar dosedependent pressor responses $[38,43]$. This pressor effect involves an increase in sympathetic nerve activity, synaptic inhibition of the baroreflex in the nucleus of the tractus solitarius, and the release of vasopressin (AVP) into the bloodstream [12].

In addition, Ang III, applied by microiontophoresis, induces higher firing rates than Ang II in the subfornical organ [44] and the hypothalamic paraventricular nucleus (PVN) [45]. Furthermore, a push-pull cannula study revealed that $93 \%$ of the angiotensin material released in the PVN following simulation with veratridine or water deprivation was in the form of Ang III [46]. Using specific and selective APA (EC33) and APN (EC27 or PC18) inhibitors, we showed that brain Ang III was one of the main effector peptides of the brain RAS in the control of AVP release and vasopressinergic neuron activity $[42,47,48]$.

In an attempt to evaluate the respective roles of brain Ang II and Ang III in the control of BP, Batt et al. [14] first evaluated the central effects of the aminopeptidase inhibitors amastatin and bestatin on BP. ICV treatment with amastatin or bestatin alone induced robust pressor responses in conscious rats that were blocked by previously ICV treatment with $\left[\mathrm{Sar}^{1}{ }^{1} \mathrm{Thr}^{8}\right]$ Ang II (sarthran), an angiotensin receptor antagonist. Thus, the pressor responses induced by these inhibitors were mediated by the brain RAS, but this did not differentiate between Ang II and Ang III, either of which could be the effector peptide responsible for the increase in BP.

In 1999, Reaux et al. [49••] provided new information concerning this issue, by blocking each of the metabolic pathways of brain Ang II and Ang III with EC33 and PC18. Previous central treatment with the APA inhibitor EC33 blocked the pressor effect of ICV Ang II in anesthetized normotensive WKY rats and SHR, suggesting that the increase in BP requires the conversion of Ang II to Ang III [49••]. Furthermore, the inhibition of endogenous brain Ang III formation by ICV, but not by intravenous (IV) injection of EC33 alone, induced a large dose-dependent decrease in BP in conscious SHRs. This hypotensive action of EC33 was also observed in another experimental model of hypertension: the DOCA-salt rat $[32 \bullet \bullet, 50]$, which is a salt- and volume-dependent but renin-independent (low plasma-renin levels) model of hypertension that is resistant to systemic RAS blockers [51].

Evidence that Ang III is the effector peptide of the brain RAS is provided by the pressor effect induced by the APN inhibitor PC18, administered alone, by ICV injection, in normotensive rats or SHR. This hypertensive response is blocked by previous treatment with an $\mathrm{AT}_{1}$ receptor antagonist, losartan (but not with PD 123319, an $\mathrm{AT}_{2}$ receptor antagonist), thus demonstrating that blocking the action of APN on Ang III metabolism leads to an increase in brain endogenous Ang III levels, resulting in an increase in BP, through interaction with $\mathrm{AT}_{1}$ but not $\mathrm{AT}_{2}$ receptors. Finally, the complete inhibition by EC33 of the PC18-induced increase in BP [49••] confirms the existence of the endogenous enzymatic cascade (see Fig. 2).

Consistent with these data, Wright et al. [16] showed that, despite the high molecular mass of APA and APN ( 120-130 kDa for the monomer), the ICV infusion of APA results in a significant increase in $\mathrm{BP}$, whereas the ICV injection of APN into SHR decreases BP [52]. The hypertensive effect probably results from higher levels of brain Ang III production, whereas the hypotensive effect may be related to an increase in Ang III metabolism. In addition, ICV infusion of an antiserum that inhibited APA activity reduced the Ang II-induced BP increase by 59\% [53].

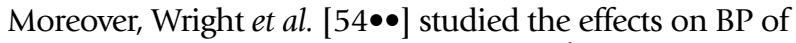
the metabolism-resistant analogs D-Asp ${ }^{1}$ Ang II and DArg ${ }^{1}$ Ang III injected ICV into conscious normotensive rats in the presence and absence of EC33 and PC18. They also concluded that Ang III was a centrally active ligand of the brain RAS in the control of BP. This underlines the fact that selectively blocking the formation of brain Ang III results in a 


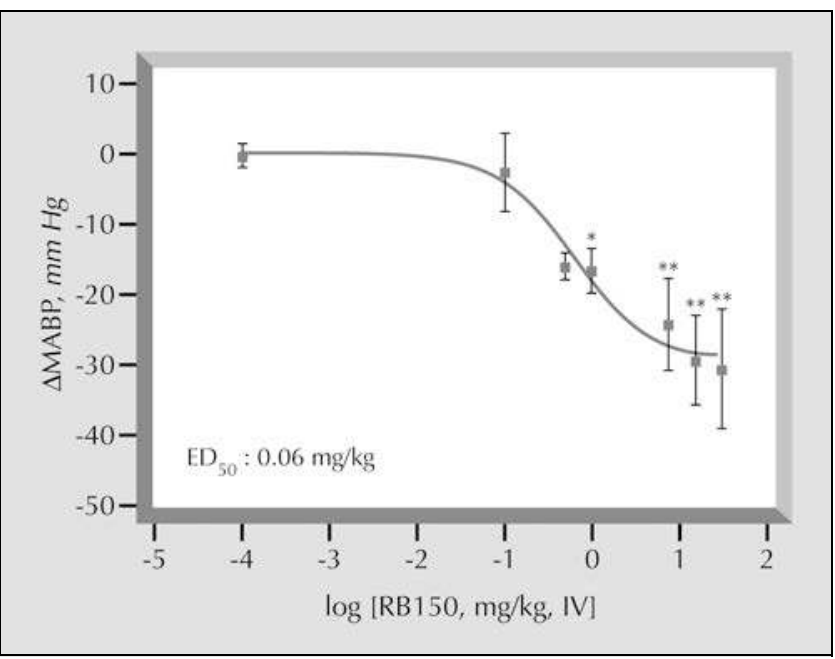

Figure 3. Effects of RB150 on MABP in conscious DOCA-salt rats. Dose response curve of MABP after IV injection of RB150 in conscious DOCA-salt rats. Mean \pm SEM variation in MABP following IV injection of RB150 $(0.1,0.5,1.0,7.5,15.0$, and $30.0 \mathrm{mg} / \mathrm{kg})$ in conscious DOCA-salt rats ( $n=5$ for each dose). ${ }^{*} P<0.05 ;{ }^{* *} P<0.01$ versus variation in MABP values obtained with the same IV dose of RB150 in conscious sham rats. DOCA—deoxycorticosterone acetate; IV—intravenous; MABP—-mean arterial blood pressure; SEM—standard error of the mean.

decrease in BP. The enzyme that generates Ang III may, therefore, be a possible target for the treatment of hypertension.

If APA inhibitors are to be used as central antihypertensive agents, they must be able to cross the blood brain barrier and inhibit brain APA activity when given by the systemic route. The central bioavailability of thiol-inhibitors of zinc metallopeptidases such as NEP or APN [30] is strongly enhanced by designing prodrugs obtained by dimerizing the compound through disulfide bridge formation. This was achieved with EC33, yielding a systemically active APA inhibitor prodrug, RB150. As the thiol group of RB150 is engaged in a disulfide bridge, it is unable to interact with the zinc atom present in the active site of APA $[18,24 \bullet \bullet, 30]$. However, in vivo the disulfide bridge of the prodrug can be cleaved by brain reductases [30], generating the active inhibitor EC33. In this respect, the IV injection of RB150 in DOCA-salt rats induces a rapid and significant decrease in brain APA activity $(62 \%)[32 \bullet \bullet] 1$ hour after the injection, demonstrating the ability of RB150 to cross the blood brain barrier.

From a series of experiments, we showed that $1.8 \%$ of the prodrug penetrates into the brain. Thus, the IV administration of $15 \mathrm{mg} / \mathrm{kg}$ of RB150 corresponds to the presence in the brain of $220 \mathrm{nmol}$ of the prodrug, which, after disulfide cleavage, completely blocks the conversion of brain Ang II to Ang III and results in a decrease of arterial BP of $30 \mathrm{~mm} \mathrm{Hg}$ (Fig. 3).

The hypotensive effect of RB150 is dose-dependent (Fig. 3B) and long-lasting, because at a dose close to the $\mathrm{ED}_{50}$ value, the reduction in $\mathrm{BP}$ is still significant 24 hours

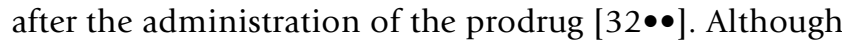

the amplitude of the hypotensive effect is high, heart rate is not modified (not shown), suggesting that the baroreflex is inhibited by brain Ang III, in agreement with the study of Lin et al. [55].

Our data show that the systemic administration of RB 150 blocks brain RAS activity and consequently

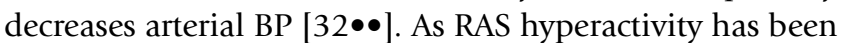
observed in DOCA-salt rat brain, resulting in increased AVP release [56] and increased sympathetic neuron activity [57], this could account for the high efficiency with which APA inhibitors decreased BP in this model.

Finally, the participation of APA to the conversion of Ang II into Ang III at the periphery remains unclear. Previous studies [58] using bestatin and amastatin did not support the conclusion that APA is a component of the systemic RAS. However, current experiments by our laboratory, in rats treated with EC33 intravenously, showed a very limited blockade of the conversion of Ang II into Ang III in the blood circulation (Hus-Citharel et al., Unpublished data) compared with an almost total inhibition after ICV EC33 treatment [42].

This discrepancy can be explained if 1) APA is not the main enzyme responsible for the conversion of Ang II into Ang III at the periphery; and 2) systemic Ang III is more rapidly degraded than brain Ang III. Therefore, inhibition of systemic Ang III formation is undetectable even after systemic APA inhibition. This could explain the minor role of circulating Ang III in the control of BP in spite of its high affinity for $\mathrm{AT}_{1}$ receptors. 3) Alternative degradation pathways of systemic Ang II other than APA exist. Such pathways would explain the absence of systemic Ang IIinduced BP increases following IV injection of EC33. In this context, the recent report by Mitsui et al. [59] showing a slight elevation of systolic BP in APA genetically deficient mice cannot be easily explained. However, on one hand, the mechanism by which APA deficiency results in hypertension was not addressed in that article, and on the other hand, one must consider that the chronic inactivation of APA achieved in APA-deficient mice cannot be easily compared with an acute blockade of this enzyme, as discussed earlier. Indeed, the total absence of APA during fetal and adult life could elicit compensatory mechanisms leading to this slight hypertensive effect, which is not contradictory with our hypothesis that APA plays a major role in the conversion of Ang II to Ang III in the brain, whereas this role appears minor at the periphery.

\section{Conclusions}

Exploration of the APA active site and the development of selective and specific APA inhibitors have made it possible to demonstrate that Ang III, generated by APA, is a major effector peptide of the central RAS in the control of AVP release and arterial BP. Brain Ang III exerts a tonic stimulatory effect on arterial BP in conscious hypertensive animals. Therefore, the inhibition of central, but not 
peripheral, APA with specific and selective inhibitors leads to a decrease in BP. Thus, central APA constitutes an interesting candidate target for the treatment of hypertension. This justifies the development of systemically active APA inhibitors, such as RB150, to inhibit brain APA activity, to block the formation of brain Ang III, and to decrease arterial BP following IV administration. Therefore, these molecules could be used as the prototype of a new class of antihypertensive agents for the treatment of certain forms of hypertension and may be particularly beneficial in hypertensive patients with low plasma renin and high plasma AVP levels, who are resistant to the usual antihypertensive medication [60].

\section{References and Recommended Reading}

Papers of particular interest, published recently, have been highlighted as:

- Of importance

$\bullet \quad$ Of major importance

1. Oparil S, Chen Y-F, Berecek KH, et al.: The role of the central nervous system in hypertension. In Hypertension: Pathophysiology, Diagnosis and Management. Edited by Laragh JH, Brenner BM. New York: Raven Press; 1995:713-740.

2. Basso N, Ruiz P, Mangiarua E, Taquini AC: Renin-like activity in the rat brain during the development of DOCA-salt hypertension. Hypertension 1981, 3:II-14-17.

3. Ganten D, Herman K, Bayer C, et al.: Angiotensin synthesis in the brain and increased turnover in hypertensive rats. Science 1983, 221:869-871.

4. Senanayake PD, Moriguchi A, Kumagai H, et al.: Increased expression of angiotensin peptides in the brain of transgenic hypertensive rats. Peptides 1994, 15:919-926.

5. Averill DB, Matsumura K, Ganten D, Ferrario CM: Role of area postrema in transgene hypertension. Hypertension 1996, 27:591-597.

6. Davisson RL, Yang G, Beltz TG, et al.: The brain renin-angiotensin system contributes to the hypertension in mice containing both the human renin and human angiotensinogen transgenes. Circ Res 1998, 83:1047-1058.

7. Morimoto S, Cassell MD, Beltz TG, et al.: Elevated blood pressure in transgenic mice with brain-specific expression of human angiotensinogen driven by the glial fibrillary acidic protein promoter. Circ Res 2001, 89:365-372.

8. Allen AM, Paxinos G, Song KF, Mendelsohn FAO: Localization of angiotensin receptor binding sites in the rat brain. In Handbook of Chemical Neuroanatomy, vol 11: Neuropeptide Receptors in the CNS. Edited by Björklund A, Hökfelt T, Kuhar MJ. Amsterdam: Elsevier; 1992:1-37.

9. Lenkei Z, Palkovits M, Corvol P, Llorens Cortes C: Expression of angiotensin type-1 (AT1) and type-2 (AT2) receptor mRNAs in the adult rat brain: a functional neuroanatomical review. Front Neuroendocrinol 1997, 18:383-439.

10. Murphy TJ, Alexander RW, Griendling KK, et al.: Isolation of a cDNA encoding the vascular type-1 angiotensin II receptor. Nature 1991, 351:233-236.

11. Mukoyama M, Nakajima M, Horiuchi $M$, et al.: Expression cloning of type 2 angiotensin II receptor reveals a unique class of seven-transmembrane receptors. J Biol Chem 1993, 268:24539-24542.

12. Phillips MI: Functions of angiotensin in the central nervous system. Annu Rev Physiol 1987, 49:413-435.

13. Saavedra JM: Brain and pituitary angiotensin. Endocr Rev 1992, 13:329-380.
14. Batt CM, Klein EW, Harding JW, Wright JW: Pressor responses to amastatin, bestatin and Plummer's inhibitors are suppressed by pretreatment with the angiotensin receptor antagonist sarthran. Brain Res Bull 1988, 21:731-735.

15. Wright JW, Jensen LL, Roberts KA, et al.: Structure-function analyses of brain angiotensin control of pressor action in rats. Am J Physiol 1989, 257:R1551-1557.

16. Wright JW, Mitzutani S, Murray CE, et al.: Aminopeptidaseinduced elevation and reduction in blood pressure in the spontaneously hypertensive rat. J Hypertens 1990, 8:969-974.

17. Wang JY, Cooper MD: A histidine residue in the zinc-binding motif of aminopeptidase $A$ is critical for enzymatic activity. Proc Natl Acad Sci U S A 1993, 90:1222-1226.

18. Vazeux G, Wang J, Corvol P, Llorens-Cortes C: Identification of glutamate residues essential for catalytic activity and zinc coordination in aminopeptidase A. J Biol Chem 1996, 271:9069-9074.

19. Vazeux G, Iturrioz X, Corvol P, Llorens-Cortes C: A tyrosine residue essential for catalytic activity in aminopeptidase A. Biochem J 1997, 327:883-889.

20. Dion N, LeMoual H, Crine P, Boileau G: Kinetic evidence that His-711 of neutral endopeptidase 24.11 is involved in stabilization of the transition state. FEBS Lett 1993, 318:301-304.

21. Beaumont A, O'Donohue MJ, Paredes N, et al.: The role of histidine 231 in thermolysin-like enzymes. J Biol Chem 1995, 270:16803-16808.

22. Vazeux G, Iturrioz X, Corvol P, Llorens-Cortes C: A glutamate residue contributes to the exopeptidase specificity in aminopeptidase A. Biochem J 1998, 334:407-413.

23. Luciani N, Marie-Claire C, Ruffet $\mathrm{E}$, et al.: Characterization of Glu350 as a critical residue involved in the $\mathrm{N}$ - terminal amine binding site of aminopeptidase N (EC 3.4.11.2): insights into its mechanism of action. Biochemistry 1998, 37:686-692

24.• Rozenfeld R, Iturrioz X, Maigret B, Llorens-Cortes C: Contribution of molecular modeling and site-directed mutagenesis to the identification of two structural residues, Arg-220 and Asp-227, in aminopeptidase A. J Biol Chem 2002, 277:29242-29252.

This is the first three-dimentional model of a strict monozinc aminopeptidase, aminopeptidase A. This model constitutes a new tool in designing inhibitors of this enzyme.

25. Rozenfeld R, Iturrioz X, Okada M, et al.: Contribution of molecular modeling and site-directed mutagenesis to the identification of a new residue, glutamate 215 , involved in the exopeptidase specificity of aminopeptidase A. Biochemistry 2003, 42:14785-14793.

26. Thunnissen MM, Nordlund P, Haeggstrom JZ: Crystal structure of human leukotriene $A(4)$ hydrolase, a bifunctional enzyme in inflammation. Nat Struct Biol 2001, 8:131-135.

27. Wilk S, Thurston LS: Inhibition of angiotensin III formation by thiol derivatives of acidic amino acids. Neuropeptides 1990 , 16:163-168

28. Roques BP, Noble F, Daugé V, et al.: Neutral endopeptidase 24.11: structure, inhibition, and experimental and clinical pharmacology. Pharmacol Rev 1993, 45:87-145.

29. Chauvel EN, Coric P, Llorens-Cortes C, et al.: Investigation of the active site of aminopeptidase $A$ using a series of new thiol-containing inhibitors. J Med Chem 1994, 37:1339-1346.

30. Fournie-Zaluski MC, Coric P, Turcaud S, et al.: Potent and systemically active aminopeptidase $\mathrm{N}$ inhibitors designed from active-site investigation. J Med Chem 1992, 35:1259-1266.

31. Reaux A, de Mota N, Zini S, et al.: PC18, a specific aminopeptidase $\mathrm{N}$ inhibitor, induces vasopressin release by increasing the half-life of brain angiotensin III. Neuroendocrinology 1999, 69:370-376.

32.• Fournie-Zaluski MC, Fassot C, Valentin B, et al.: Brain reninangiotensin system blockade by systemically active aminopeptidase A inhibitors: a potential treatment of saltdependent hypertension. Proc Natl Acad Sci U S A 2004, 101:7775-7780.

This study describes the antihypertensive action of a new systemically active APA inhibitor RB150 in DOCA-salt rats. 
33. Malfroy B, Kado-Fong H, Gros C, et al.: Molecular cloning and amino acid sequence of rat kidney aminopeptidase $\mathrm{M}$ : a member of a super family of zinc-metallohydrolases. Biochem Biophys Res Commun 1989, 161:236-241.

34. Wu Q, Lahti JM, Air GM, et al.: Molecular cloning of the murine BP-1/6C3 antigen: a member of the zinc-dependent metallopeptidase family. Proc Natl Acad Sci 1990, 87:993-997.

35. Wilk S, Healy DP: Glutamyl aminopeptidase (aminopeptidase A), the BP-1/6C3 antigen. Adv Neuroimmunol 1993, 3:195-207.

36. Palmieri FE, Bausback HH, Ward PE: Metabolism of vasoactive peptides by vascular endothelium and smooth muscle aminopeptidase M. Biochem Pharmacol 1989, 38:173-180.

37. Harding JW, Yoshida MS, Dilts RP, et al:: Cerebroventricular and intravascular metabolism of [125I] angiotensins in rat. $J$ Neurochem 1986, 46:1292-1297.

38. Abhold RH, Sullivan MJ, Wright JW, Harding JW: Binding, degradation and pressor activity of angiotensins II and III after aminopeptidase inhibition with amastatin and bestatin. $J$ Pharmacol Exp Ther 1987, 242:957-962.

39. Harding JW, Felix D: The effects of aminopeptidase inhibitors amastatin and bestatin on angiotensin-evoked neuronal activity in rat brain. Brain Res 1987, 424:299-304.

40. Tieku S, Hooper NM: Inhibition of aminopeptidases N, A and $\mathrm{W}$ : a re-evaluation of the actions of bestatin and inhibitors of angiotensin converting enzyme. Biochem Pharmacol 1992, 44:1725-1730.

41. Checler F: Neuropeptide-degrading peptidases. In Methods in Neurotransmitter and Neuropeptide Research, vol 13. Edited by Parvez SH, Naoi M, Nagatsu T. Amsterdam: Elsevier Science; 1993:375-418

42. Zini S, Fournie-Zaluski MC, Chauvel E, et al.: Identification of metabolic pathways of brain angiotensin II and III using specific aminopeptidase inhibitors: predominant role of angiotensin III in the control of vasopressin release. Proc Natl Acad Sci U S A 1996, 93:11968-11973.

43. Wright JW, Morseth SL, Abhold RH, Harding JW: Pressor action and dipsogenicity induced by angiotensin II and III in rats. Am J Physiol 1985, 249:R514-R521.

44. Felix D, Schlegel W: Angiotensin receptive neurones in the subfornical organ: structure-activity relations. Brain Res 1978, 149:107-116.

45. Harding JW, Felix D: Angiotensin-sensitive neurons in the rat paraventricular nucleus: relative potencies of angiotensin II and angiotensin III. Brain Res 1987, 410:130-134.

46. Harding JW, Jensen LL, Hanesworth JM, et al.: Release of angiotensins in paraventricular nucleus of rat in response to physiological and chemical stimuli. Am J Physiol 1992, 262:F17-F23.

47. Zini S, Demassey Y, Fournie-Zaluski MC, et al.: Inhibition of vasopressinergic neurons by central injection of a specific aminopeptidase A inhibitor. Neuroreport 1998, 9:825-828.
48. Reaux A, Fournie-Zaluski MC, Llorens-Cortes C: Angiotensin III: a central regulator of vasopressin release and blood pressure. Trends Endocrinol Metab 2001, 12:157-162.

49.• Reaux A, Fournie-Zaluski MC, David C, et al.: Aminopeptidase A inhibitors as potential central antihypertensive agents. Proc Natl Acad Sci U S A 1999, 96:13415-13420.

This article reports evidence that brain Ang III exerts a stimulatory effect on $\mathrm{BP}$ in conscious spontaneously hypertensive animals.

50. Iturrioz X, Reaux-Le Goazigo A, Llorens-Cortes C: Aminopeptidase inhibitors as anti-hypertensive drugs. In Aminopeptidases in Biology and Disease. Edited by Lendeckel U, Hooper N. New York: Kluwer-Plenum; 2004:229-250.

51. Morton JJ, Casals-Stenzel J, Lever AF, et al.: Inhibitors of the renin-angiotensin system in experimental hypertension, with a note on the measurement of angiotensin I, II and III during infusion of converting-enzyme inhibitor. $\mathrm{Br} J$ Clin Pharmacol 1979, 7:233S-241S

52. Wright JW, Jensen LL, Cushing LL, Harding JW: Leucine aminopeptidase $\mathrm{M}$-induced reductions in blood pressure in spontaneously hypertensive rats. Hypertension 1989, 13:910-915.

53. Song L, Wilk S, Healy DP: Aminopeptidase A antiserum inhibits intracerebroventricular angiotensin II-induced dipsogenic and pressor responses. Brain Res 1997, 744:1-6.

$54 . \bullet$ Wright JW, Tamura-Myers E, Wilson WL, et al.: Conversion of brain angiotensin II to angiotensin III is critical for pressor response in rats. Am J Physiol Regul Integr Comp Physiol 2003, 284:R725-R733.

Conversion of brain angiotensin II to angiotensin III is critical for pressor response in rats, and Ang III's importance as a centrally active ligand has been underestimated.

55. Lin KS, Chan JY, Chan SH: Involvement of AT2 receptors at NRVL in tonic baroreflex suppression by endogenous angiotensins. Am J Physiol 1997, 272:H2204-H2210.

56. Nishimura M, Ohtsuka K, Sakamoto M, et al.: Roles of brain angiotensin II and C-type natriuretic peptide in deoxycorticosterone acetate-salt hypertension in rats. J Hypertens 1998, 16:1175-1185.

57. Masuyama Y, Tsuda K, Kuchii M, Nishio I: Peripheral neural mechanism of hypertension in rat models-peripheral sympathetic neurotransmission in hypertension. J Hypertens Suppl 1986, 4:S189-S192.

58. Ahmad S, Ward PE: Role of aminopeptidase activity in the regulation of the pressor activity of circulating angiotensins. $J$ Pharmacol Exp Ther 1990, 252:643-650.

59. Mitsui $T$, Nomura $S$, Okada $M$, et al:: Hypertension and angiotensin II hypersensitivity in aminopeptidase A-deficient mice. Mol Med 2003, 9:57-62.

60. Bakris G, Bursztyn M, Gavras I, et al.: Role of vasopressin in essential hypertension: racial differences. J Hypertens 1997, 15:545-550 